\title{
Metabolic Syndrome and Outcomes after Renal Intervention
}

\author{
Daynene Vykoukal ${ }^{1,2}$ and Mark G. Davies ${ }^{1,2}$ \\ ${ }^{1}$ Department of Cardiovascular Surgery, Methodist DeBakey Heart and Vascular Center, \\ The Methodist Hospital, 6550 Fannin, Smith Tower, Suite 1401, Houston, TX 77030, USA \\ ${ }^{2}$ Vascular Biology and Therapeutics Program, The Methodist Hospital Research Institute, Houston, TX 77030, USA \\ Correspondence should be addressed to Mark G. Davies, mdavies@tmhs.org
}

Received 14 October 2010; Revised 27 November 2010; Accepted 10 December 2010

Academic Editor: Ken Ichi Aihara

Copyright $\odot 2011$ D. Vykoukal and M. G. Davies. This is an open access article distributed under the Creative Commons Attribution License, which permits unrestricted use, distribution, and reproduction in any medium, provided the original work is properly cited.

\begin{abstract}
Metabolic syndrome significantly increases the risk for cardiovascular disease and chronic kidney disease. The increased risk for cardiovascular diseases can partly be caused by a prothrombotic state that exists because of abdominal obesity. Multiple observational studies have consistently shown that increased body mass index as well as insulin resistance and increased fasting insulin levels is associated with chronic kidney disease, even after adjustment for related disorders. Metabolic syndrome appears to be a risk factor for chronic kidney disease, likely due to the combination of dysglycemia and high blood pressure. Metabolic syndrome is associated with markedly reduced renal clinical benefit and increased progression to hemodialysis following endovascular intervention for atherosclerotic renal artery stenosis. Metabolic syndrome is associated with inferior early outcomes for dialysis access procedures.
\end{abstract}

\section{Introduction}

Metabolic syndrome is evolving into a pandemic, contributing to approximately 6-7\% for all-cause mortality, $12-$ $17 \%$ for cardiovascular disease, and $30-52 \%$ for diabetes in the population [1]. In populations free of cardiovascular disease at baseline, cardiovascular morbidity and mortality increases 1.5 - to 3 -fold in the presence of the metabolic syndrome $[2,3]$. Two definitions of metabolic syndrome predominate the literature, the National Cholesterol Education Program (NCEP) and the World Health Organization (WHO). Metabolic syndrome is defined as the presence of 3 or more of the following: (1) waist circumference $\geq 88 \mathrm{~cm}$ in women and $\geq 102 \mathrm{~cm}$ in men, (2) fasting triglycerides $\geq 150 \mathrm{mg} / \mathrm{dL}$ or drug treatment for elevated triglycerides, (3) HDL-cholesterol $<50 \mathrm{mg} / \mathrm{dL}$ in women and $<40 \mathrm{mg} / \mathrm{dL}$ in men or drug treatment for reduced HDL-cholesterol, (4) $\mathrm{BP} \geq 130 / 85 \mathrm{mmHg}$ or use of BP-lowering medication, and (5) fasting glucose $\geq 100 \mathrm{mg} / \mathrm{dL}$ or use of glucoselowering medication, according to the American Heart Association/National Heart, Lung, and Blood Institute (AHA/NHLBI), updated National Cholesterol Education Program (NCEP), and Adult Treatment Panel III (ATPIII) criteria [4]. If waist circumference is not available, a body mass index (BMI) $>30 \mathrm{~kg} / \mathrm{m}^{2}$ can be used as a determinant for abdominal obesity [5]. Metabolic syndrome appears to be a risk factor for chronic kidney disease, likely due to the combination of dysglycemia, a prothrombotic state and high blood pressure. This paper examines the relationship between metabolic syndrome and the development of and interventions for renal artery and renal parenchymal disease.

\section{Coagulation}

The increased risk for cardiovascular diseases could partly be caused by a prothrombotic state that exists because of abdominal obesity. Adipose tissue induces thrombocyte activation by the dysregulated production of adipose tissuederived hormones, often called adipokines, which have been shown to interfere with platelet function. Enhanced platelet aggregation and activation markers are associated with low adiponectin concentrations [6]. Increased adipose tissue mass induces insulin resistance and systemic low-grade inflammation, also affecting platelet function. It has been demonstrated that adipose tissue directly impairs fibrinolysis 
by the production of plasminogen activator inhibitor-1 and possibly thrombin-activatable fibrinolysis inhibitor [7]. Adipose tissue may contribute to enhanced coagulation by direct tissue factor production, but hypercoagulability is likely to be primarily caused by altered hepatic synthesis of the coagulation factors fibrinogen, factor VII, factor VIII, and tissue factor, by releasing free fatty acids and proinflammatory cytokines (tumor necrosis factor- $\alpha$, interleukin$1 \beta$, and interleukin-6) into the portal circulation and by inducing hepatic insulin resistance. Adipose tissue dysfunction could thus play a causal role in the prothrombotic state observed in obesity, by directly and indirectly affecting hemostasis, coagulation, and fibrinolysis [8]. Platelets in type 2 diabetic individuals adhere to vascular endothelium and aggregate more readily than those in healthy people. Loss of sensitivity to the normal homeostatic restraints exercised by prostacyclin $\left(\mathrm{PGI}_{2}\right)$ and nitric oxide (NO) generated by the vascular endothelium presents as the major defect in platelet function. Insulin is a natural antagonist of platelet hyperactivity. It sensitizes the platelet to $\mathrm{PGI}_{2}$ and enhances endothelial generation of $\mathrm{PGI}_{2}$ and NO. Thus, the defects in insulin action in diabetes create a milieu of disordered platelet activity conducive to macrovascular and microvascular events. Patients with type 2 diabetes and abdominal fat patterning displayed higher plasma activities of clotting factors VII and VIII as well as increased plasma levels of fibrinogen and von Willebrand factor antigen, when compared with not only healthy normal weight controls, but also with diabetic patients at normal body weight. An altered coagulation state has implications for any surgical or endovascular intervention.

\section{Obesity and Kidney Disease}

Obesity and the metabolic syndrome significantly increase the risk for cardiovascular disease and chronic kidney disease. Multiple abnormalities that can lead to kidney injury have been identified in overweight and obese people, including insulin resistance, compensatory hyperinsulinemia, inappropriate activation of the renin-angiotensinaldosterone system, increased oxidative stress, endoplasmic reticulum stress, coagulability, and impaired fibrinolysis. The combined effects of these conditions induce, in the kidneys, impaired pressure natriuresis, glomerular hypertension, endothelial dysfunction, and vasoconstriction, as well as matrix proliferation and expansion. Among the consequences are microalbuminuria, now known to be a surrogate of diffuse endothelial dysfunction as well as a predictor of cardiovascular disease, and chronic kidney disease [9]. Multiple observational studies have consistently shown that increased body mass index as well as insulin resistance and increased fasting insulin levels is associated with chronic kidney disease, even after adjustment for related disorders [10]. Obesity may promote intracellular lipid accumulation in the kidney. Prevalence of a body mass index of at least $35 \mathrm{~kg} / \mathrm{m}^{2}$ among incident dialysis patients has increased by $64 \%$ over the past decade, and if these trends continue $20 \%$ of all patients will initiate dialysis with this degree of obesity. Weight loss improves glomerular hemodynamics in morbidly obese adults and may retard progression of chronic kidney disease. In contrast, once a patient reaches endstage renal disease, the degree of adiposity correlates with survival, and weight loss may not necessarily be beneficial [11]. Metabolic syndrome appears to be a risk factor for chronic kidney disease, likely due to the combination of dysglycemia and high blood pressure. Metabolic syndrome is associated with markedly reduced renal clinical benefit and increased progression to hemodialysis following endovascular intervention for atherosclerotic renal artery stenosis.

\section{Cardiovascular Risk and Peripheral Vascular Procedures}

Patients with peripheral arterial disease and metabolic syndrome have an increased risk for the development of cardiovascular (CV) events, when compared to patients without metabolic syndrome (27\% versus $18 \%$ and $27 \%$ versus $19 \%, P<.001$, resp.). In peripheral arterial disease, metabolic syndrome was independently associated with an increased risk of long-term CV events ( $\mathrm{HR}=1.6$; 95\% CI $1.2-2.1$ and $\mathrm{HR}=1.4 ; 95 \%$ CI $1.1-1.8$ ) but not CV mortality [12]. A survey of patients with intermittent claudication and ankle/brachial index $(\mathrm{ABI})<0.90$ showed that $52.6 \%$ meet the revised version of the Adults Treatment Panel III (rATP III) criteria for metabolic syndrome [13]. In women, metabolic syndrome is associated with an increased risk of future symptomatic peripheral arterial disease. This risk appears to be mediated largely by the effects of inflammation (increased levels of high-sensitivity C-reactive protein) and endothelial activation (increased levels of soluble intercellular adhesion molecule-1). There is currently no data on the impact of metabolic syndrome on outcomes in peripheral interventions. After open aneurysm repair, obesity of any class is independently predictive of wound complications. Class III obesity was also an independent predictor of renal complications and cardiac complications. After EVAR (endovascular aortic/aneurysm repair), obesity (any class) was predictive of wound complications, but not predictive of other complications or death. For the two types of operation, there were fewer complications and deaths after EVAR compared with open repair across all BMI categories, but outcomes were most disparate among the obese [14]. Obesity is associated with extended operation time during EVAR. After controlling for age, gender, and operation type, mortality risk remained lowest in obese class I patients (odds ratio (OR) $0.63, P=.023$ ), while morbidity risk was highest in obese class III patients (OR 1.70, $P=.0003$ ), due to wound infection, thromboembolism, and renal complications [15]. Surgical site infections occur frequently after lower extremity bypass regardless of bypass origin and are associated with early graft failure and sepsis. Obesity predicts postoperative surgical site infections. Mortality risk was greatest in the underweight, followed by morbidly obese and normalweight patients, while overweight and mild to moderate obesity were associated with the lowest mortality. 


\section{Interventions and Renal Fibromuscular Dysplasia}

Hypertension is one of the criteria for metabolic syndrome and one cause of treatable hypertension in women is fibromuscular dysplasia (FMD) [16]. Percutaneous endovascular intervention for clinically symptomatic fibromuscular dysplasia in the renal arteries is technically successful and safe. There are excellent assisted patency and low restenosis rates with immediate clinical benefit for most patients and continued long-term results up to 5 years [17]. Using proportional hazard analysis, the predictors of long-term clinical benefit were duration of hypertension $<8$ years, creatinine $<1.5 \mathrm{mg} / \mathrm{dL}$, ipsilateral kidney size, functional status of the contralateral kidney, a fasting blood sugar $<110 \mathrm{mg} / \mathrm{dL}^{*}$, triglycerides $<150 \mathrm{mg} / \mathrm{dL}^{*}$, and $\mathrm{HDL}>$ $50 \mathrm{mg} / \mathrm{dL}^{*}$ (*components of metabolic syndrome) [17]. Neither age $<50$ years nor administration of statins appeared to be significant. The diagnosis of metabolic syndrome did not affect outcomes. It appears, however, that the presence of existing renal pathology and markers of metabolic syndrome is associated with recurrence of hypertensive symptoms [17].

\section{Interventions and Renal Atherosclerotic Disease}

Endovascular therapy for symptomatic atherosclerotic renal artery stenosis is common and effective in the wellselected patient $[18,19]$. The proportion of patients with symptomatic renal artery disease who will have metabolic syndrome is similar to that described for symptomatic peripheral vascular disease [20]. These patients will more often be female, but there is no significant difference in presenting symptoms. Despite the presence of obesity and the risks associated with metabolic syndrome, perioperative mortality and morbidity are equivalent to those without metabolic syndrome. Patients with metabolic syndrome have equivalent survival and cumulative patency. However, the metabolic syndrome group has a lower 5-year freedom from restenosis and lower 5-year retained clinical benefit (freedom from recurrent hypertension or worsening renal insufficiency) (Figure 1). However, a higher number of patients progress to hemodialysis (3\% versus $13 \%$, no metabolic syndrome versus metabolic syndrome; $P<.01)$. Individually, the components of metabolic syndrome do not influence outcomes [20].

\section{Dialysis Access}

The average BMI of incident dialysis patients in USA has risen by $13 \%$ since 1995 , mirroring the expanding obesity epidemic in USA. This increase in patient weight has resulted in an increased number of dialysis patients who are obese (BMI > 30). The impact of BMI on dialysis access outcomes has been difficult to elucidate, with some studies showing inferior outcomes for dialysis access procedures among obese patients, while others do not detect a BMIassociated outcome effect. When determining the route of

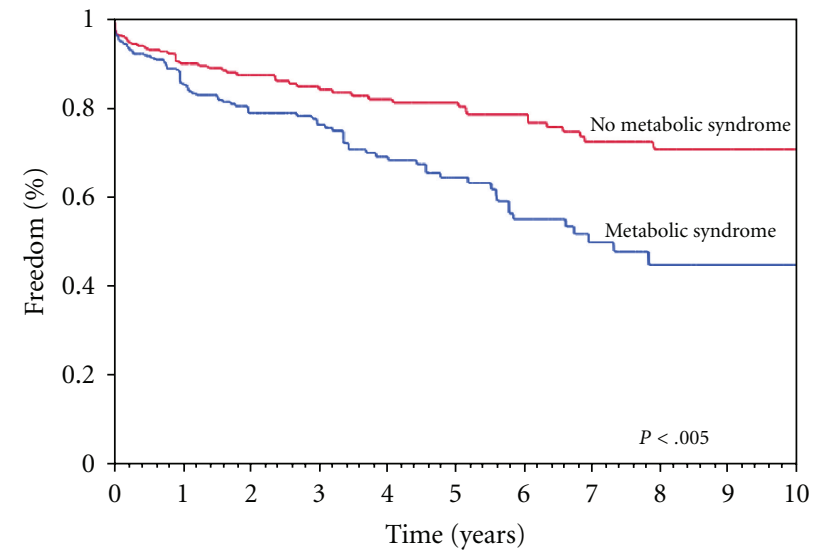

Figure 1: Kaplan-Meier analysis of freedom from renal-related morbidity (persistent increase in creatinine $>20 \%$ of baseline, progression to hemodialysis, death from renal-related causes) for the patients with and without MetS. Values are mean.

dialysis access in the obese, proper consideration must be given to peritoneal dialysis (PD). While the placement of PD catheters in obese individuals may be technically challenging, there is no decrease in catheter use or catheter survival in obese patients compared to the nonobese. Obese PD patients do have higher catheter-exit site infection rates and a tendency toward poor wound healing. Placement of autologous fistulae and prosthetic grafts in the obese follows the same guidelines as in the nonobese. However, in the case of autologous fistulae, superficialization (i.e., removing subcutaneous fat to decrease the depth of the vein and allow cannulization) is more common in these patients and will delay primary access. There is no data on the impact of metabolic syndrome on the functionality and longevity of dialysis access sites.

Metabolic syndrome influences the development of chronic renal insufficiency and does influence the outcomes of percutaneous renal intervention in patients with symptomatic atherosclerotic renal and FMD renal artery disease. In addition, the presence of obesity, as well as metabolic syndrome, alters the paradigms for obtaining dialysis access for those with end-stage renal disease and complicates the ability to achieve successful and durable access.

\section{References}

[1] E. S. Ford, "Risks for all-cause mortality, cardiovascular disease, and diabetes associated with the metabolic syndrome: a summary of the evidence," Diabetes Care, vol. 28, no. 7, pp. 1769-1778, 2005.

[2] J. K. Olijhoek, Y. Van Der Graaf, J. D. Banga, A. Algra, T. J. Rabelink, and F. L. J. Visseren, "The Metabolic Syndrome is associated with advanced vascular damage in patients with coronary heart disease, stroke, peripheral arterial disease or abdominal aortic aneurysm," European Heart Journal, vol. 25, no. 4, pp. 342-348, 2004.

[3] H. M. Lakka, D. E. Laaksonen, T. A. Lakka et al., "The metabolic syndrome and total and cardiovascular disease mortality 
in middle-aged men," Journal of the American Medical Association, vol. 288, no. 21, pp. 2709-2716, 2002.

[4] S. M. Grundy, J. I. Cleeman, S. R. Daniels et al., "Diagnosis and management of the metabolic syndrome: an American Heart Association/National Heart, Lung, and Blood Institute scientific statement," Circulation, vol. 112, no. 17, pp. 27352752, 2005.

[5] K. G. M. M. Alberti and P. Z. Zimmet, "Definition, diagnosis and classification of diabetes mellitus and its complications. Part 1: diagnosis and classification of diabetes mellitus. Provisional report of a WHO consultation," Diabetic Medicine, vol. 15, no. 7, pp. 539-553, 1998.

[6] P. Restituto, I. Colina, J. J. Varo, and N. Varo, "Adiponectin diminishes platelet aggregation and SCD40L release. Potential role in the metabolic syndrome," American Journal of Physiology - Endocrinology and Metabolism, vol. 298, no. 5, pp. E1072-E1077, 2010.

[7] Y. Aso, "Plasminogen activator inhibitor (PAI)-1 in vascular inflammation and thrombosis," Frontiers in Bioscience, vol. 12, pp. 2957-2966, 2007.

[8] D. R. Faber, PH. G. De Groot, and F. L. J. Visseren, "Role of adipose tissue in haemostasis, coagulation and fibrinolysis," Obesity Reviews, vol. 10, no. 5, pp. 554-563, 2009.

[9] G. Lastra, C. Manrique, and J. R. Sowers, "Obesity, cardiometabolic syndrome, and chronic kidney disease: the weight of the evidence," Advances in Chronic Kidney Disease, vol. 13, no. 4, pp. 365-373, 2006.

[10] P. A. Sarafidis and L. M. Ruilope, "Insulin resistance, hyperinsulinemia, and renal injury: mechanisms and implications," American Journal of Nephrology, vol. 26, no. 3, pp. 232-244, 2006.

[11] H. Kramer and A. Luke, "Obesity and kidney disease: a big dilemma," Current Opinion in Nephrology and Hypertension, vol. 16, no. 3, pp. 237-241, 2007.

[12] J. P. van Kuijk, W. J. Flu, M. Chonchol, J. J. Bax, H. J. M. Verhagen, and D. Poldermans, "Metabolic syndrome is an independent predictor of cardiovascular events in high-risk patients with occlusive and aneurysmatic peripheral arterial disease," Atherosclerosis, vol. 210, no. 2, pp. 596-601, 2010.

[13] G. Brevetti, E. Laurenzano, G. Giugliano et al., "Metabolic syndrome and cardiovascular risk prediction in peripheral arterial disease," Nutrition, Metabolism and Cardiovascular Diseases, vol. 20, no. 9, pp. 676-682, 2010.

[14] O. N. Johnson, A. N. Sidawy, J. M. Scanlon et al., "Impact of Obesity on Outcomes after Open Surgical and Endovascular Abdominal Aortic Aneurysm Repair," Journal of the American College of Surgeons, vol. 210, no. 2, pp. 166-177, 2010.

[15] D. L. Davenport, E. S. Xenos, P. Hosokawa, J. Radford, W. G. Henderson, and E. D. Endean, "The influence of body mass index obesity status on vascular surgery 30 -day morbidity and mortality," Journal of Vascular Surgery, vol. 49, no. 1, pp. 140 147, 2009.

[16] R. A. Davidson, Y. Barri, and C. S. Wilcox, "Predictors of cure of hypertension in fibromuscular renovascular disease," American Journal of Kidney Diseases, vol. 28, no. 3, pp. 334338, 1996.

[17] M. G. Davies, W. E. Saad, E. K. Peden, I. T. Mohiuddin, J. J. Naoum, and A. B. Lumsden, "The long-term outcomes of percutaneous therapy for renal artery fibromuscular dysplasia," Journal of Vascular Surgery, vol. 48, no. 4, pp. 865-871, 2008.

[18] M. G. Davies, W. A. Saad, J. X. Bismuth, E. K. Peden, J. J. Naoum, and A. B. Lumsden, "Outcomes of endoluminal reintervention for restenosis after percutaneous renal angioplasty and stenting," Journal of Vascular Surgery, vol. 49, no. 4, pp. 946-952, 2009.

[19] M. G. Davies, W. E. Saad, J. X. Bismuth, J. J. Naoum, E. K. Peden, and A. B. Lumsden, "Endovascular revascularization of renal artery stenosis in the solitary functioning kidney," Journal of Vascular Surgery, vol. 49, no. 4, pp. 953-960, 2009.

[20] M. G. Davies, W. E. Saad, J. Bismuth, J. J. Naoum, E. K. Peden, and A. B. Lumsden, "Impact of metabolic syndrome on the outcomes of percutaneous renal angioplasty and stenting," Journal of Vascular Surgery, vol. 51, no. 4, pp. 926-932, 2010. 


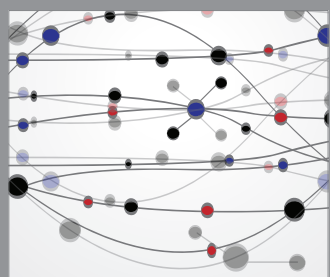

The Scientific World Journal
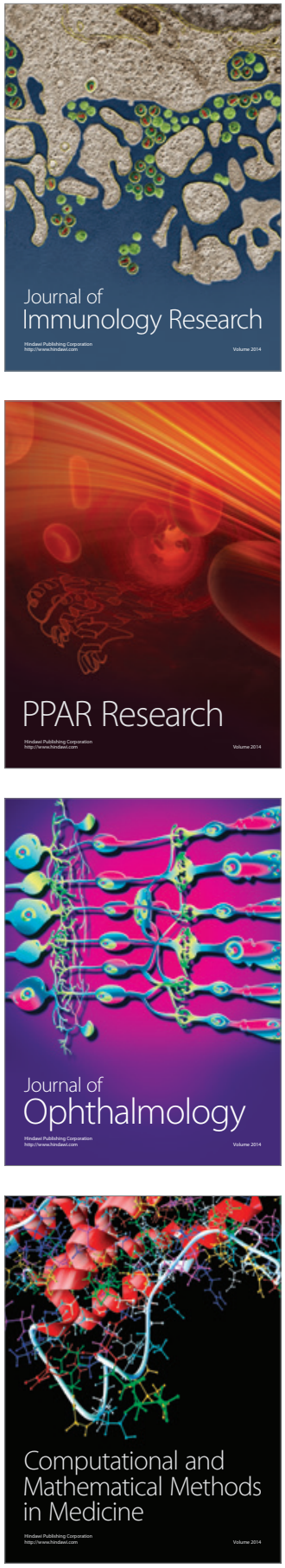

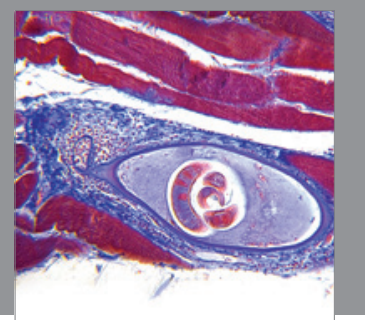

Gastroenterology

Research and Practice
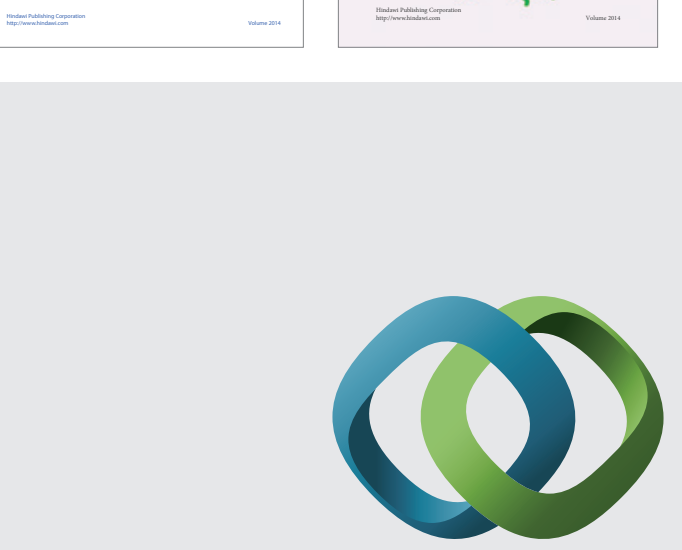

\section{Hindawi}

Submit your manuscripts at

http://www.hindawi.com
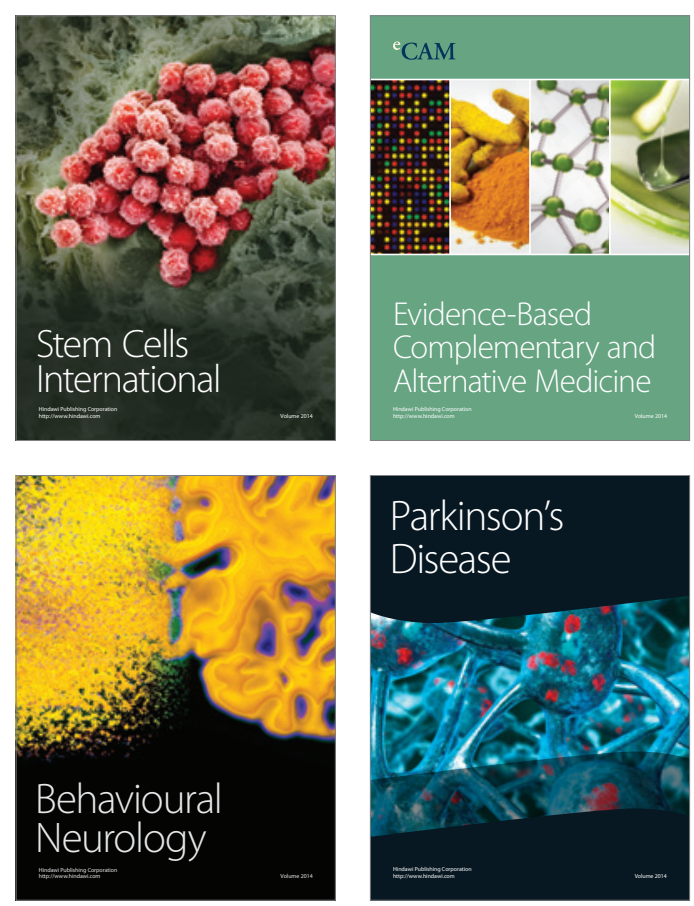

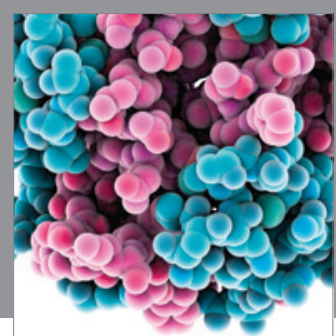

Journal of
Diabetes Research

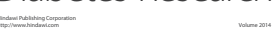

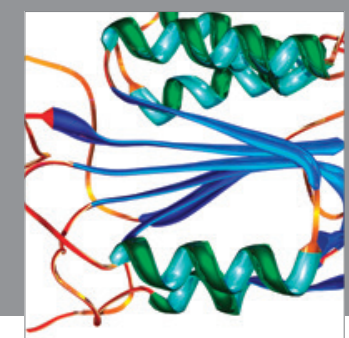

Disease Markers
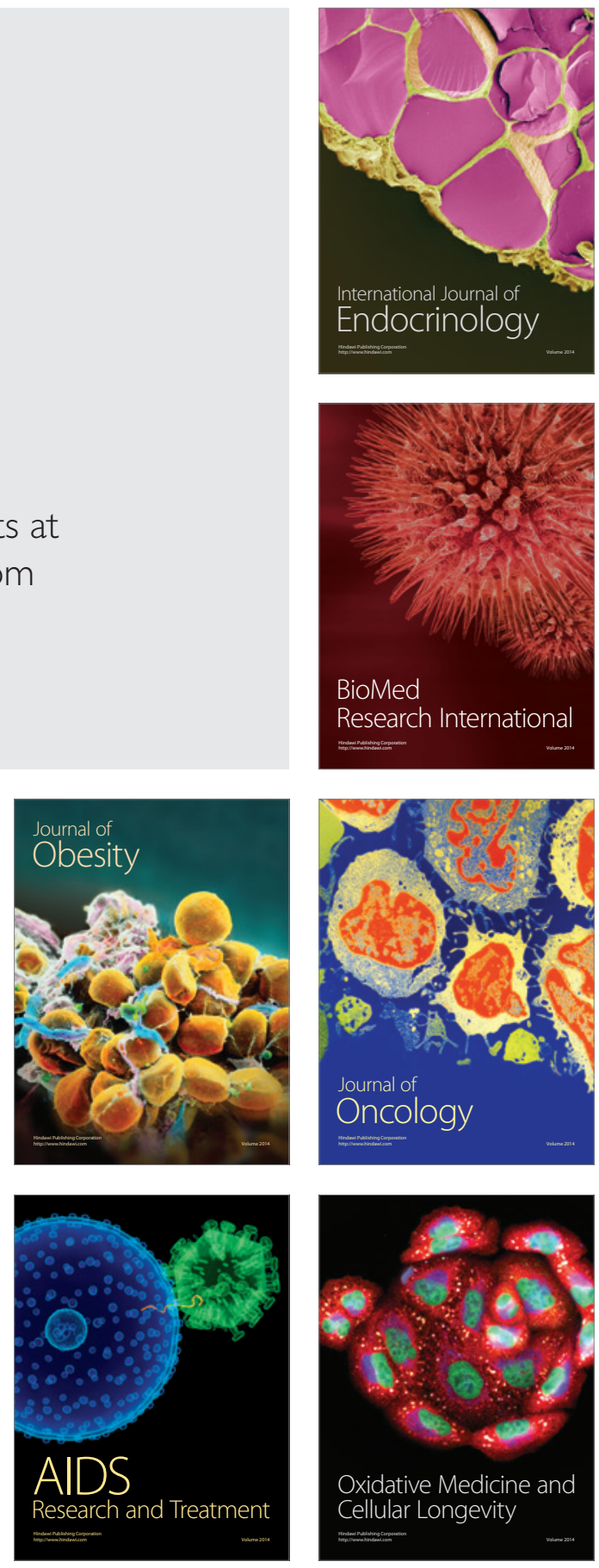